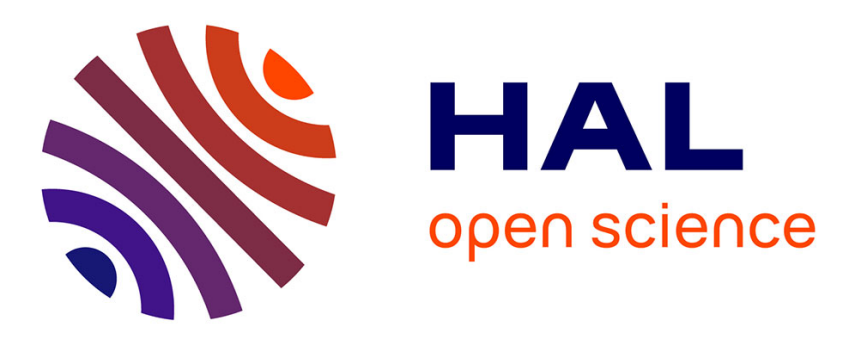

\title{
X-ray method for the structural investigation of thin organic films
}

\author{
F. Rieutord, J.J. Benattar, Louis Bosio
}

\section{To cite this version:}

F. Rieutord, J.J. Benattar, Louis Bosio. X-ray method for the structural investigation of thin organic films. Journal de Physique, 1986, 47 (7), pp.1249-1256. 10.1051/jphys:019860047070124900 . jpa00210314

\section{HAL Id: jpa-00210314 https://hal.science/jpa-00210314}

Submitted on 1 Jan 1986

HAL is a multi-disciplinary open access archive for the deposit and dissemination of scientific research documents, whether they are published or not. The documents may come from teaching and research institutions in France or abroad, or from public or private research centers.
L'archive ouverte pluridisciplinaire HAL, est destinée au dépôt et à la diffusion de documents scientifiques de niveau recherche, publiés ou non, émanant des établissements d'enseignement et de recherche français ou étrangers, des laboratoires publics ou privés. 
Classification

Physics Abstracts

$68.90-68.55-61.65$

\title{
$X$-ray method for the structural investigation of thin organic films
}

\author{
F. Rieutord, J. J. Benattar and L. Bosio $\left(^{*}\right)$ \\ DPhG/SPSRM - CEN Saclay 91191 Gif-sur-Yvette Cedex, France \\ (*) Laboratoire CNRS «Physique des Liquides et Electrochimie " associé à l'Université Pierre et Marie Curie, \\ T22, 4, Place Jussieu, 75230 Paris Cedex 05, France
}

(Reçu le 29 octobre 1985, révisé le 26 février 1986, accepté le 7 mars 1986)

\begin{abstract}
Résumé. - Nous montrons que la simple conjugaison de trois techniques conventionnelles de rayons $\mathrm{X}$ permet une étude structurale des films minces lamellaires. La diffraction en transmission du rayonnement synchrotron fournit une détermination de l'arrangement intra-lamellaire, la diffraction en réflexion à petits angles, celle de l'ordre inter-lamellaire et enfin la réflexion critique donne une mesure de la densité nécessaire à l'obtention du nombre d'unités structurales par maille. Nous présentons une illustration de cette méthode à travers l'étude d'un composé organique déposé en couches de Langmuir-Blodgett.
\end{abstract}

\begin{abstract}
The structures of thin lamellar films can be determined through a combination of three X-ray experiments using synchrotron radiation. Diffraction experiments in the transmission geometry give the intralamellar ordering, the diffraction at small glancing angles yields the interlamellar organization, and the critical reflection provides the density measurement which is required to obtain the number of structural units per cell. This method is illustrated here with the study of an organic compound deposited in Langmuir-Blodgett films.
\end{abstract}

\section{Introduction.}

There has been considerable interest in thin organic films but only few X-ray studies of their structures have been performed until now. The main reason for this lack of structural information is the rather small thickness of the film compared with that of its substrate. Because of this major difficulty no X-ray diffraction experiments in transmission have been carried out on these systems yet. One of the aims of the present paper is to demonstrate that such an experiment can be performed under specific conditions by using $\mathrm{X}$-ray synchrotron radiation. The second purpose of this work is to show that the combination of diffraction in transmission, of diffraction at small glancing angles and of X-ray critical reflection yields a new complete method for obtaining structural information on thin films. In order to illustrate this method, we shall apply it to the structural study of an organic compound deposited in a Langmuir-Blodgett (L.B.) film. Let us recall that a L.B. film consists of a multilayered system of amphiphilic molecules. This film is obtained by successive depositions of monomolecular layers onto a substrate. The molecules of such a film are first spread onto a water bath and then laterally compressed into a solid-like monolayer. The film is then transferred by dipping the substrate through this compressed monolayer. By renewing this process in the opposite direction a second layer is deposited tail-to-tail onto the first layer and so on for the next layers. Thus this technique allows the construction of numerous interesting physical systems by varying the number of layers as well as the nature of the chemical components. A general survey of this field is given in reference [1].

The system under study is a L.B. film of a charge transfer complex which is initially an insulator labelled precursor; after iodization it becomes a conductor. We shall determine the structures of both states. After a short description of the samples, we shall describe each of the X-ray experiments successively. In the last section we shall propose a structure consistent with all the experimental data.

\section{Chemicals.}

The compound under study which has been synthetized by A. Ruaudel-Teixier, is the complex [N-docosylpyridinium, tetracyano-quino-dimethane 
(TCNQ)] whose formula is :
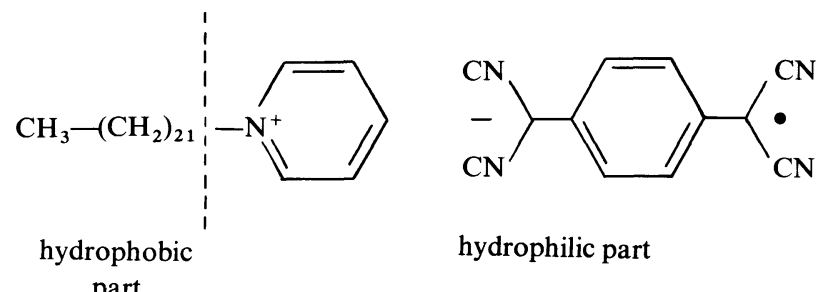

hydrophilic part

This system has been first extensively investigated by Barraud et al. [2] using UV and IR spectroscopy and conductivity measurements. They have shown that after deposition into a L.B. film, it is an insulator (precursor). In a second stage the sample is oxidized in situ by iodine vapour and this leads to a final stable conducting state. The conductivity is due to a partial oxidation of $\mathrm{TCNQ}^{-}$into $\mathrm{TCNQ}_{0}$ thus giving rise to a mixed valence complex. Therefore, the conductivity $\sigma\left(\sigma \sim 0.1 \Omega^{-1} \mathrm{~cm}^{-1}\right)$ occurs inside the polar planes, which are separated by aliphatic sublayers acting as insulators.

It should also be pointed out that, in the conducting state, iodine appears as $\mathrm{I}_{\mathbf{3}}^{-}$ions, with an unknown stoichiometry.

The diffraction in transmission experiments and the reflectivity measurements require specific samples. These different samples (number of deposited layers and nature of the substrate) used in each technique will be described further in the text. In each case the sample is studied under both forms, precursor and conductor.

\section{Diffraction experiments.}

2.1 TRANSMISSION EXPERIMENTS USING SYNCHROTRON RADIATION. - The investigation of the molecular ordering within the lamellar planes is possible only when the scattering vector

$$
\mathbf{Q}=\mathbf{k}_{\mathbf{F}}-\mathbf{k}_{\mathrm{I}}\left(\left|\mathbf{k}_{\mathrm{F}}\right|=\left|\mathbf{k}_{\mathrm{I}}\right|=\frac{2 \pi}{\lambda}\right)
$$

lies in the $\left(\mathbf{a}^{*}, \mathbf{b}^{*}\right)$ reciprocal plane corresponding here to the (a, b) plane of the layers. Thus, in such a geometry (Fig. 1), the beam passes through the

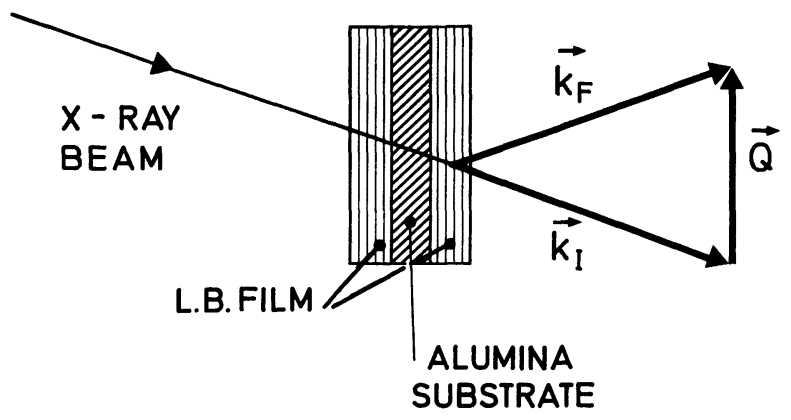

Fig. 1. - Schematic transmission geometry. substrates, whose strong diffraction signal usually, overwhelms that of the film. The standard procedure to cope with this problem is to use free-standing films [3]. In our case, such a solution cannot be used [4] since the substrate is essential to build up the layers. It is however possible to get around this difficulty by using as a substrate a very thin and weakly absorbing film; we used in our experiments a $1000 \AA$-thick film of alumina braced onto a flat ring, which has proved to yield a negligible X-ray diffusion and only one Bragg reflection clearly distinguishable.

The X-ray diffraction experiments have been performed at LURE-DCI (France) on the D16 spectrometer because of its very high flux and good resolution. We used a transmission device with a monochromatic convergent beam $(\lambda=1.759 \AA)$ selected by a $\mathrm{Ge}(111)$ single bent monochromator.

As the L.B. films are very thin (from 20 to 40 layers), the scattered intensity is very weak. Thus, to improve the signal-to-noise ratio, the whole diffraction camera is maintained under vacuum.

The high intensity of the synchrotron radiation enabled us to obtain high quality diffraction patterns. Both precursor and conductor have been investigated; their diffraction patterns are reported respectively in figures $2 \mathrm{a}$ and $2 \mathrm{~b}$. They both exhibit numerous and homogeneous sharp rings. It should be pointed out that our samples have important misorientations $\left(\lesssim 15^{\circ}\right)$ resulting from both the lack of planeity of the alumina membrane and the L.B. film deposition. Due to this effect there is a great extension of the hko rings mainly along the c-direction. Furthermore due to the thickness of the sample (14 layers), the corresponding extension $\left(c^{*} / 14\right)$ is negligible compared to the previous effect. Thus the extended $h k o$ rings always intersect the Ewald sphere and then can be observed on the diagrams. In the following we shall label the rings with $h k$ indexes. Some simple conclusions can be drawn in the light of these experiments. First, the existence of rings gives an evidence of a polycrystallinity within the layers. The size of the domains, given by the width of the rings, ranges from about $1000 \AA$ to $3000 \AA$. When the sample is rotated about an axis perpendicular to the beam, few $h k l$ rings appear (Fig. 2c); this clearly means that the layers are rather well correlated, forming a threedimensional ordered structure. In figure 3 we present a schematic drawing illustrating the macroscopic structure. It shows the layered arrangement and the microdomains within the layers separated, by grain boundaries. It should be noted that in order to improve the quality of the sample, six layers of behenic acid have been first deposited onto the substrate. Thus the X-ray patterns of both conductor and precursor exhibit a ring at $4.15 \AA$, characteristic of these first layers.

The two patterns (Figs. 2a and 2b) appear to be rather different since some of the $h k$ rings have a stronger intensity and are displaced due to the addi- 

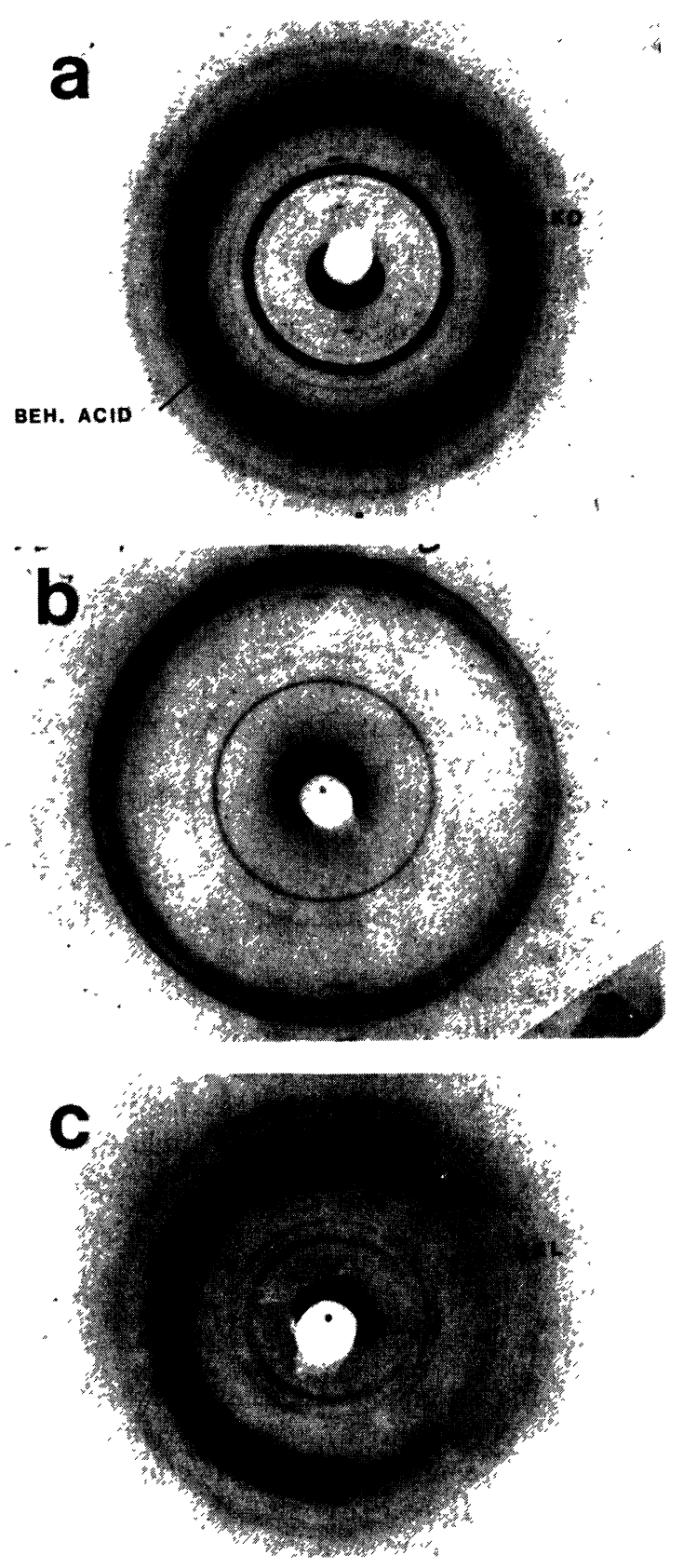

Fig. 2. - X-ray patterns performed in a transmission geometry. a) $\left(\mathbf{a}^{*}, \mathbf{b}^{*}\right)$ reciprocal plane in the precursor ; b) $\left(\mathbf{a}^{*}, \mathbf{b}^{*}\right)$ reciprocal plane in the conductor; c) pattern obtained when the sample is rotated about an axis perpendicular to the beam.

tion of iodine. The in-plane molecular arrangement is strongly distorted by iodine whose main effect is to change the TCNQ arrangement.

The indexation of the set of $h k$ reflections is given in table I for the precursor and table II for the conductor.

Our interpretation has been chosen as simple as possible but it must be noticed that the rather small number of reflections does not allow us to exclude some other more complex indexations. Nevertheless,

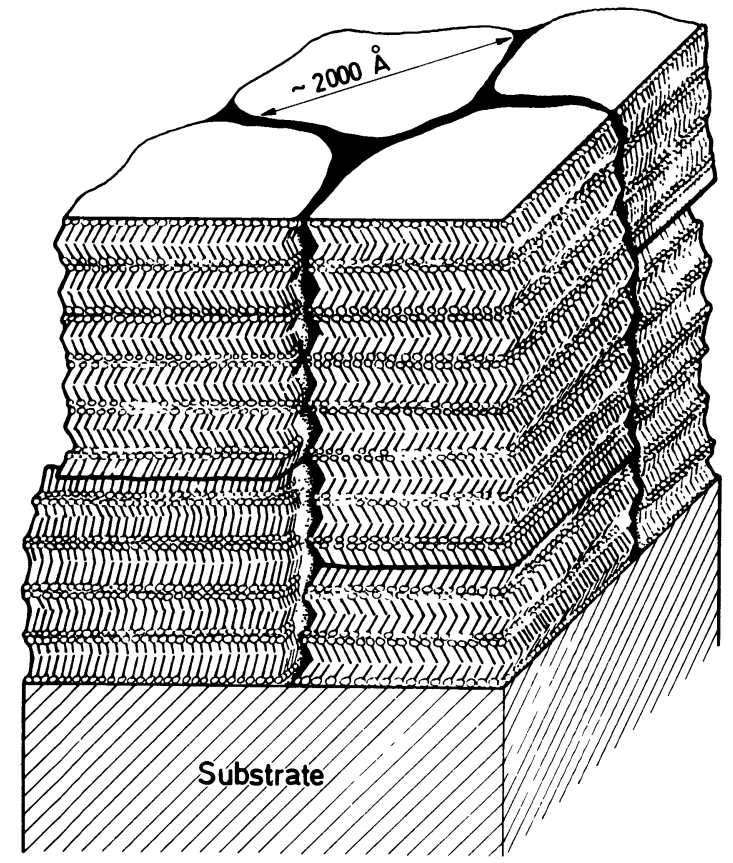

Fig. 3. - Schematic view of the microcrystalline structure.

Table I. - Indexation of the hk rings in the precursor.

\begin{tabular}{|c|c|c|}
\hline$d_{\text {exp }}(\AA)$ & $h k$ & $d_{\text {calc }}(\AA)$ \\
\hline 10.19 & 02 & 10.20 \\
7.68 & 12 & 7.75 \\
6.78 & 03 & 6.79 \\
6.32 & 20 & 6.32 \\
5.90 & 13 & 5.86 \\
5.50 & $2 \frac{3}{2}$ & 5.51 \\
4.56 & 23 & 4.52 \\
3.99 & $3 \frac{2}{3}$ & 3.98 \\
3.69 & 33 & 3.68 \\
3.55 & 30 & 3.51 \\
3.21 & 40 & 3.17 \\
\hline
\end{tabular}

Table II. - Indexation of the hk rings in the conductor.

\begin{tabular}{|c|c|c|}
\hline$d_{\exp }(\AA)$ & $h k$ & $d_{\text {calc }}(\AA)$ \\
\hline 7.70 & $1 \overline{1}$ & 7.70 \\
\hline 6.42 & $\left\{\begin{array}{l}01 \\
1\end{array}\right.$ & $\left\{\begin{array}{l}6.42 \\
\end{array}\right.$ \\
\hline 5.75 & 20 & 5.75 \\
\hline 459 & $\{11$ & $\{4.62$ \\
\hline 4.59 & $3 \overline{1}$ & 4.59 \\
\hline & $\left\{\begin{array}{l}2 \overline{2} \\
20\end{array}\right.$ & $\left\{\begin{array}{l}3.85 \\
2.2\end{array}\right.$ \\
\hline 3.84 & $3 \underline{0}$ & 3.83 \\
\hline 3.69 & $1 \overline{2}$ & 3.66 \\
\hline 3.25 & 02 & 3.21 \\
\hline
\end{tabular}


it should be pointed out that our simple interpretation is in good agreement with all the sterical and chemical data of this system.

In both cases we found an oblique basis whose parameters are : $a=12.8 \AA, b=20.4 \AA$ and $\gamma=93^{\circ}$ for the precursor, and : $a=13.8 \AA, b=7.7 \AA$ and $\gamma=123.5^{\circ}$ for the conductor.

2.2 DifFRACTION EXPERIMENTS AT SMALL GLANCING ANGLES. - The determination of the lamellar spacing and of the molecular ordering in the direction of the layer normal is usually provided by small angle diffraction experiments. In the case of L.B. films deposited onto a thick substrate, the reflection geometry is required. This geometry is schematically described in figure 4. This kind of investigation has been performed previously by B. Belbeoch et al., their results concerning the complex studied here are reported in reference [5]. From this study we will mention here the main experimental data only. The lamellar spacing in the precursor which is given by the 001 Bragg reflection only, has been found to be : $c=46 \AA$ whereas in case of the conductor, the same parameter which has been obtained from a larger number of $00 l$ Bragg reflections, is : $c=48.7 \AA$. The values are greater than the molecular length in its streched conformation $(41 \AA)$. This has been interpreted in a model involving chain interdigitation [5]. Unfortunately the in-plane parameters $(\mathbf{a}, \mathbf{b})$ given in this reference do not agree with our parameters. Indeed, the only inter-reticular distance measured is not necessarily a cell parameter. Furthermore the second parameter has not been measured but was inferred from standard distances between TCNQ. Thus, the angle $\gamma$ between $(\mathbf{a}, \mathbf{b})$ is certainly not equal to $90^{\circ}$. As far as the density $\rho$ is concerned, their experimental device using a photographic method with a convergent beam is unsuited to reflectivity measurements.

In the following, we shall not tackle the question of the aliphatic medium. In the case of the conductor, the Patterson function provides straight away the location of the heavy iodine ions. This gives the thickness of the polar sublayer which is about $18 \AA$.

\section{X-ray critical reflection.}

The knowledge of the cell parameters is not enough to propose a structural model. At this level a density measurement is required to determine the number of

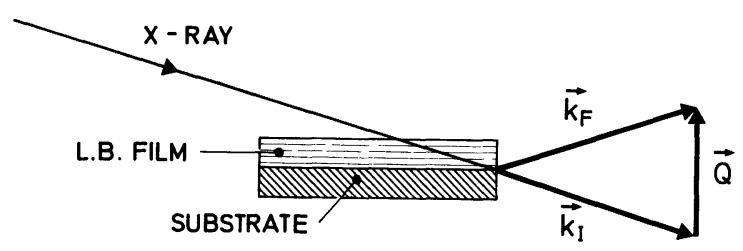

Fig. 4. - Schematic geometry of the X-ray diffraction at small angles. molecular units per cell and also the stoichiometry of the conductor. This will be provided by X-ray critical reflection. We have measured the angular dependence of the reflectivity by using an experimental device described in reference [6]. A schematic drawing of this device appears in figure 5 .

It is well known that in the range of X-ray wavelengths, the refractive index $n$ of condensed matter is less than unity and can be written as :

$$
n=1-\delta-i \beta \text {. }
$$

$\delta=C \rho$ is essentially dependent on the density $\rho$ and $C$ is nearly a constant given by

$$
C=\frac{e^{2} \mathcal{N}}{2 \pi m c^{2}} \frac{\left(f+\Delta f^{\prime}\right)}{M} \lambda^{2}
$$

$\lambda$ is the wavelength $\left(\lambda\left(\mathrm{CuK} \alpha_{1}\right)=1.5406 \AA\right), e^{2} / m c^{2}$ is the classical electron radius, $\mathcal{N}$ is the Avogadro constant, $M$ the molar mass, $f$ is the atomic scattering factor and $\Delta f^{\prime}$ is the real part of the anomalous dispersion.

In our case $\Delta f^{\prime}$ is negligible and $f$ can be approximated by the atomic number $Z$ since the reflection angle $\theta$ is small.

The imaginary part in equation (1), $\beta$, proportional to the linear absorption coefficient $\mu(\beta=\mu \lambda / 4 \pi)$; expresses the absorption of X-rays in the medium. The theory of X-ray reflectivity is based upon the Fresnel equations. For a glancing angle lower than the critical angle $\theta_{\mathrm{c}}$, the reflectivity $R(\theta)=\left(\frac{I(\theta)}{I_{0}}\right)$ is high. $\theta_{\mathrm{c}}$ is given by the relation $\sin \left(\frac{\pi}{2}-\theta_{\mathrm{c}}\right)=1-\delta$ and thus we have $\theta_{\mathrm{c}} \sim \sqrt{2 \delta}$. Note that the incident beam is totally reflected when $\beta=0$.

Thus the measurement of the angular dependence of the reflectivity from a surface provides directly both the density and the linear absorption coefficient. Then knowing the density and the cell-volume $V$, the number of molecular units $N$ is given by : $N=$ $\rho V \mathcal{N} / M$.

The measurements which have been performed on the precursor and the conductor samples are

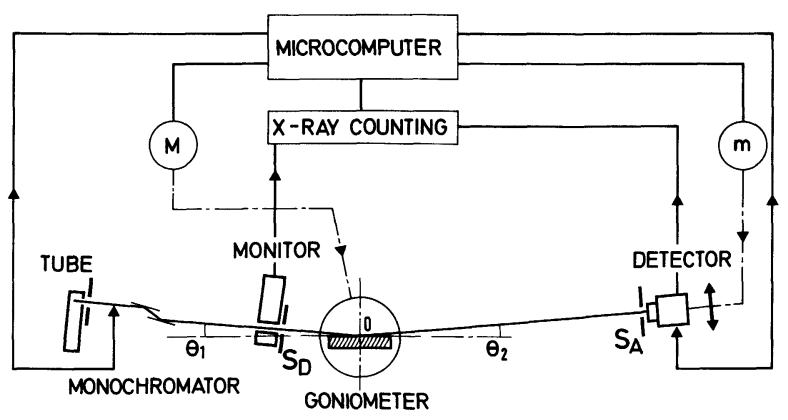

Fig. 5. - Experimental device for reflectivity measurements: the screw mechanism driven by the motor $\mathbf{M}$, acts on $\theta_{1}$ and $\theta_{2}:$ the motor moves $\theta_{2}$ via the $\mathrm{S}_{\mathrm{A}}$-detector. 


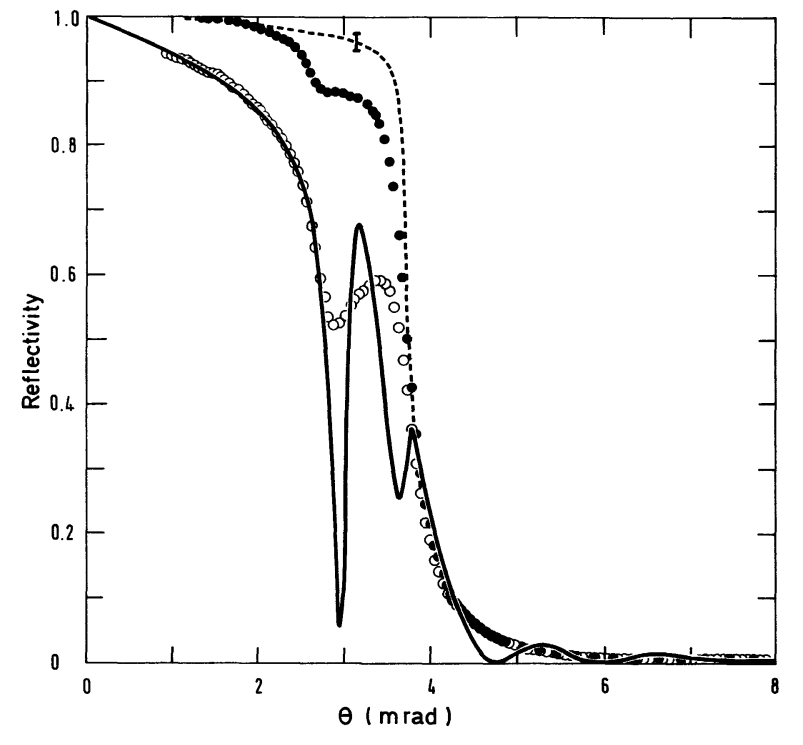

Fig. 6. - Reflectivity profiles $(\lambda(\mathrm{CuK} \alpha), 1.54 \AA)$ of the precursor (filled circles), the conductor (open circles) and the bare substrate (dotted line). The angular resolution(FWHM) is $\sim 0.2 \mathrm{mrad}$ and the accuracy of the angle determination is $\sim 0.05 \mathrm{mrad}$. The solid line curve which corresponds to a simple model using step like profiles for the three different media, is indicative of the critical angles. This model exhibits Kiessig fringes which do not appear in the experiment curve due to a lack of homogeneity of the multilayer film.

reported in figure 6. Let us recall that the precursor sample consists of a 14-layer film $(\sim 340 \AA$ \& $)$ deposited on 6 layers of behenic acid (160 $\AA$ ). The substrate was, in this case, an optical quality quartz slide $\left(6 \times 2.5 \mathrm{~cm}^{2}\right)$. The same sample is transformed after oxidation by iodine into a conductor sample.

As the film is very thin, the beam is weakly absorbed for a glancing angle $\theta$ greater than $\theta_{\mathrm{c}}$, and then the reflection on the quartz substrate can be observed on the reflectivity curves (Fig. 6). It should be pointed out that there are no Kiessig fringes resulting from interferences betwen the beams reflected at the two main interfaces, air/film and film/substrate. This suggests a lack of uniformity in the thickness of the multilayer film which may be due to some collapses of the layers.

To determine the parameters $\delta$ and $\beta$ of equation (1) for X-ray reflected intensity, the density profile (i.e. the variation of the refractive index as a function of the $z$-coordinate along the layer normal) must be known. The expressions for such a profile, which have been proposed by Nevot et al. [7], take into account the rugosity and the transition zone between two adjacent media so involving several parameters. In the present case, we have simply assumed step-like profiles and then the only two parameters $\delta$ and $\beta$ of this medium had to be fitted, since layer thicknesses were given (the values of $\delta$ and $\beta$ of both behenic acid and quartz are well-known data).
Owing to the small thickness of this film and the very low X-ray absorption of the precursor, the accuracy of the determination of $\delta_{\mathrm{p}}$ and $\beta_{\mathrm{p}}$ is in this case rather poor

$$
\begin{gathered}
\delta_{\mathrm{p}}=3.4 \pm 0.9 \times 10^{-6} \\
7 \times 10^{-9}<\beta_{\mathrm{p}}<7 \times 10^{-8} .
\end{gathered}
$$

In this range of corresponding values of the number of molecular units per cell, some other physical considerations lead us to choose $N=16$ for a cell volume $V=12 \times 10^{3} \AA^{3}$.

In the case of the conductor, the model using steplike profiles does not completely fit the experimental curve. Indeed this model exhibits Kiessig fringes whereas they do not appear in the experimental data due to a lack of homogeneity of the film. Nevertheless this simple model allows the determination of the absorption and the critical angles. We found :

$$
\begin{aligned}
& \delta_{\mathrm{c}}=4.1 \pm 0.2 \times 10^{-6} \\
& \beta_{\mathrm{c}}=3.2 \pm 0.2 \times 10^{-7} .
\end{aligned}
$$

It should be mentioned that the accuracy is much greater due to the higher absorption of this iodized sample. The above values provide the density and the linear absorption coefficient of the conductor :

$\rho_{\mathrm{c}}=1.23 \pm 0.06 \mathrm{~g} / \mathrm{cm}^{2}$ and $\mu_{\mathrm{c}}=260 \pm 15 \mathrm{~cm}^{-1}$.

From the knowledge of $\mu_{\mathrm{c}}$ it is easy to deduce the quantity of iodine in the sample. Since iodine has been incorporated as $\mathrm{I}_{3}^{-}$ions, we found that the stoichiometry is approximately : one $\mathrm{I}_{3}^{-}$ion for three molecular units. Thus the number of molecular units per cell, consistent with the density $\rho_{\mathrm{c}}$, appears to be $N=4$ for a volume $V=4310 \AA^{3}$.

\section{Structural models.}

As has been shown in the previous sections, X-ray techniques easily provide the main part of the data required in order to build a structural model. The cell parameters and the number of molecular units per cell being known, steric considerations as well as other chemical properties give complementary information which enable us to propose structures for the precursor and the conductor.

4.1 Precursor sample. - Let us now gather the corresponding set of results. First we found a monoclinic cell whose parameters are : $a=12.8 \AA$, $b=20.4 \AA, c=46 \AA$ and $\gamma=93^{\circ}$ with a number of molecular units $N=16$.

In figure 7 we have shown the TCNQ arrangement of the polar sublayer in the oblique basis of the cell.

As the $a$ parameter corresponds exactly to 4 times the standard distance $(3.2 \AA)$ between TCNQ planes [8], it is obvious that the lateral stacking of the TCNQ lies along the a-axis. It should be mentioned that some 


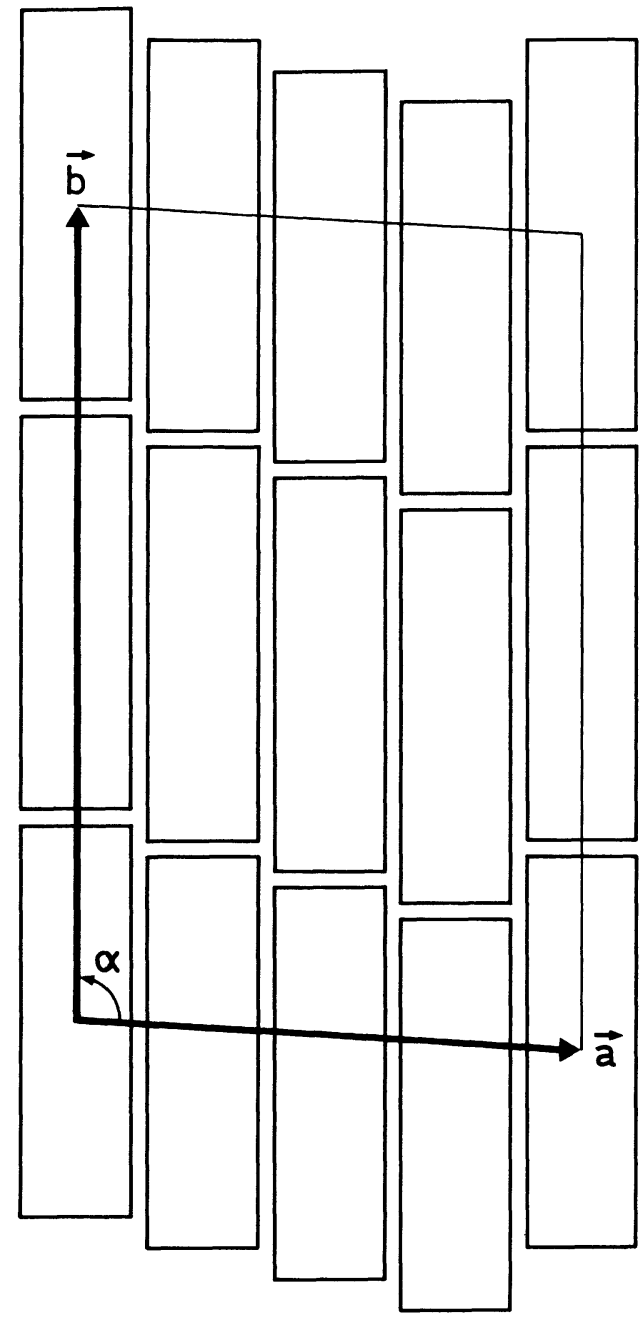

Fig. 7. - Projection on the oblique basis of the monoclinic cell in the precursor showing the TCNQ arrangement.

dimerization of the TCNQ has been observed [2] which has only a weak influence on the lateral stacking of TCNQ. This dimerization related to the 4-unit periodicity has been also evidenced in a similar compound (TMA-TCNQ salt) [8].

The $b$ parameter appears to be a little less than twice the length of the TCNQ in its long axis. This implies that this long axis may form a small angle $\omega$ with the $(\mathbf{a}, \mathbf{b})$ plane $\left(\omega \sim 10\right.$ to $\left.15^{\circ}\right)$. As far as the pyridinium rings are concerned, their position can be easily determined knowing that there are electrostatic interactions with the $\mathrm{TCNQ}^{-}$. Thus due to a closepack of the TCNQ, it appears that the pyridinium rings are located just above the TCNQ and are coplanar with them.

Figure 8 gathers all the structural features relative to the precursor and shows an overall view of the structure consistent with all the experimental data. Let us emphasize that structure extends only inside each microdomain whereas the macroscopic structure should be viewed as represented in figure 3 .

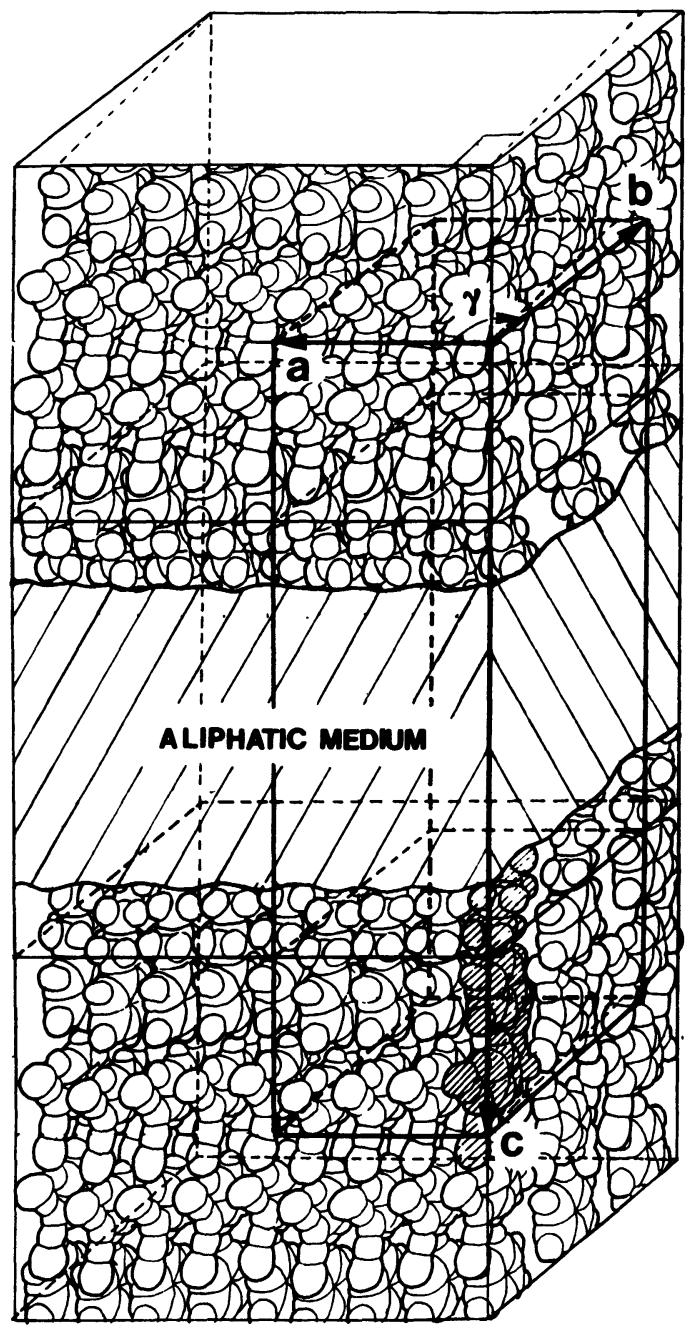

Fig. 8. - A perspective view of the molecular packing in the monoclinic cell of the precursor.

4.2 CONDUCTOR SAMPLE. - This sample is the final stage of the oxido-reduction process and the most interesting since it is a stable 2D-organic conductor. The iodine stoichiometry of this conductor has been measured, as well as the density, by reflectivity experiments which are here much more accurate than in the case of the precursor. Let us bring together the results of X-ray measurements; the parameters of the monoclinic cell were found to be : $a=13.8 \AA, b=7.7 \AA$, $c=48.7 \AA$ and $\gamma=123.5^{\circ}$. The iodine stoichiometry and the number of molecular units are as follows : 4 units [TCNQ $\left.{ }^{-}, \mathrm{CH}_{3}\left(\mathrm{CH}_{2}\right)_{21} \mathrm{Py}^{+}\right]$and $4 / 3 \mathrm{I}_{3}^{-}$per cell. We recall that small angle diffraction [5] yields the distance between the $\mathrm{I}_{3}^{-}$ions. They are located on both sides of the polar sublayer, separated by $18 \AA$ and probably beside the $\mathrm{Py}^{+}$ions.

The TCNQ arrangement in the $(\mathbf{a}, \mathbf{b})$ plane is drawn in figure 9. As far as the $a$ parameter is concerned, it appears that the only modification is due to a slight restacking of the TCNQ. On the contrary the bdirection is much more altered in this restacking and 


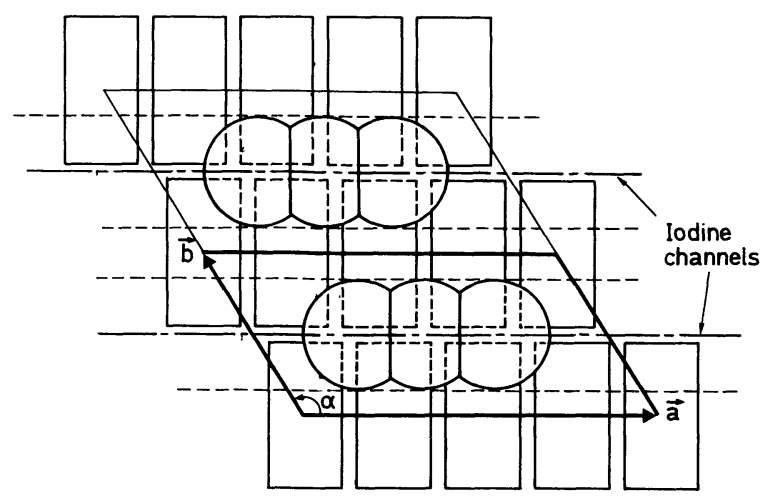

Fig. 9. - Projection on the oblique basis of the monoclinic cell in the conductor showing the TCNQ arrangement and the iodine channels.

the orthogonal projection of the $b$-parameter corresponds now to the length of the small axis of the TCNQ molecules. The main modification of the structure consists in the setting-up of the long TCNQ axis and the regular stacking of the TCNQ planes.

In these organic conductors, there is one-dimensional conductivity which is due to electron-delocalization along the stacking direction of TCNQ. Since the microcrystalline domains are oriented at random around the layer normal, this leads to a 2Dconductivity, the conducting polar planes being isolated from one another by the aliphatic medium. The steric hindrance of the $\mathrm{I}_{3}^{-}$ions imposes them to be aligned within channels parallel to the a-direction. In figure 10, we propose a perspective view of the structure of the conductor gathering all the experimental data.

\section{Conclusion.}

In thin solid films studies, it is clear that any physical investigation should be preceded by a complete structural study of the system. Until now only few structural parameters (i.e. the layer spacing mainly) could be measured by X-ray diffraction. We have shown in this paper that the sole use of X-ray techniques allows also the determination of the complementary parameters. For the first time we have performed high flux diffraction experiments in transmission using very thin films of alumina as substrate thus giving directly access to the in-plane order. Note however that electron diffraction in transmission is also a suitable method for this kind of investigation $[9,10]$. In addition to these experiments, reflectivity measurements have been achieved to get the essential parameters, density and absorption linear coefficient.

The conjunction of these $\mathrm{X}$-ray techniques provides a new tool which, including the knowledge of chemical data, enables a complete investigation of the structure.

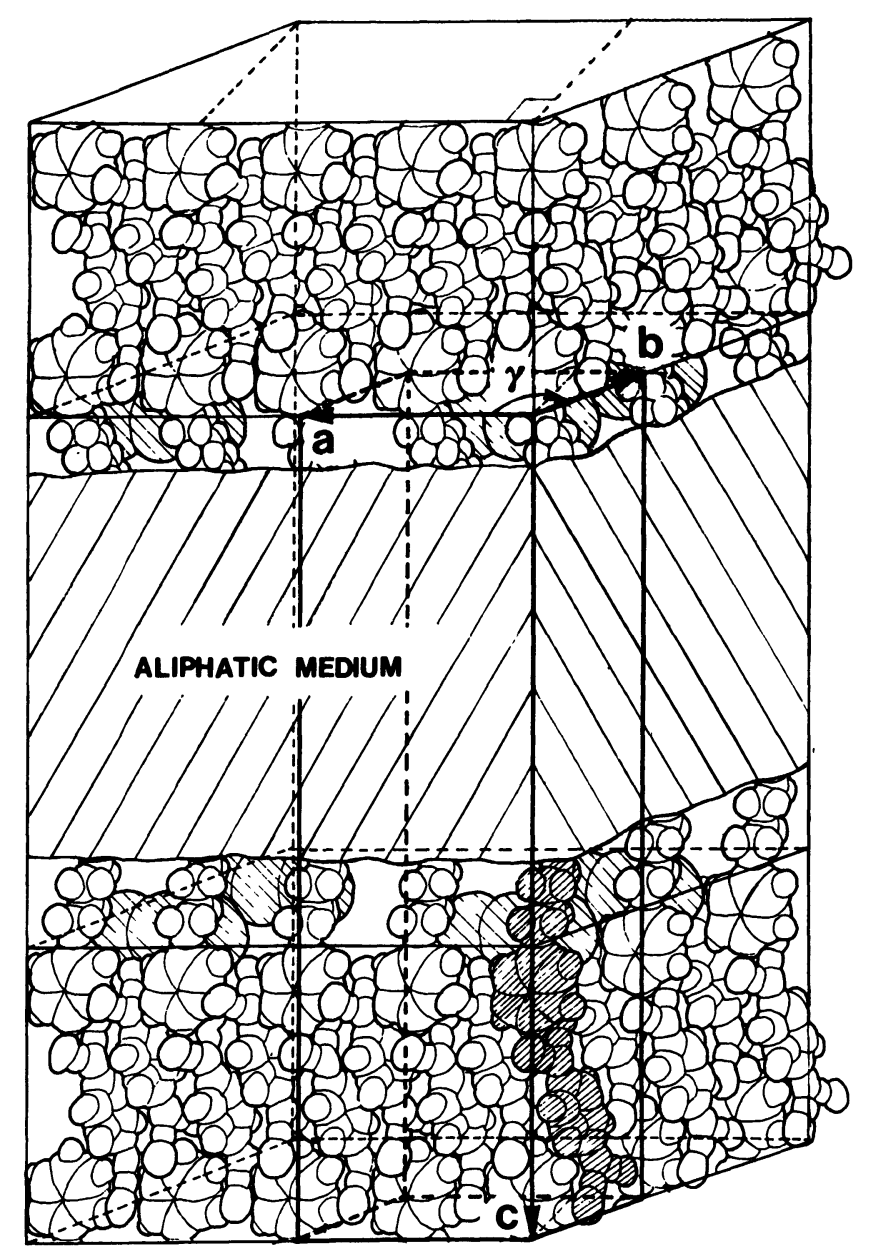

Fig. 10. - A perspective view of the molecular packing in the monoclinic cell of the conductor.

Applying this method to the case of a charge transfer complex deposited in L.B. films, we have shown the existence of a two-dimensional polycrystallinity which makes this system a 2D-conductor. We have proposed structural models for both samples precursor and conductor but these models have their limitations due to the great complexity of this ternary compound.

Using simpler compounds it will be possible with this method to carry out experimental studies on new physical problems like for example phase transitions in L.B. films.

\section{Acknowledgments.}

We wish to thank A. Barraud who initiated the work on this system, J. Richard, A. Ruaudel-Teixier and M. Vandevyver for the samples, B. Belbeoch, M. Roulliay and M. Tournarie for information about small angle diffraction, S. Megtert for synchrotron diffraction experiments at LURE and R. de Kouchkovsky for helpful discussions. 


\section{References}

[1] Thin Solid Film 99, no 1/2/3 (1983).

[2] Ruaudel-Teixier, A., Barraud, A., Vandevyver, M., Belbeoch, B., Roulliay, M., C.R. Soc. Fr. Chim. Congrès Nancy (1984).

[3] Pindak, R., Moncton, D. E., Davey, S. C. and GoodBY, J. W., Phys. Rev. Lett. 46 (1981) 1135.

[4] Prakash, M., Dutta, P., Ketterson, J. B. and Abraham, B. M., Chem. Phys. Lett. 111 (1984) 395.

[5] Belbeoch, B., Roulliay, M. and Tournarie, M., 2nd International Congress on L.B. films Schenectady (1985), to be published in Thin Solid Films.
[6] Bosio, L., Cortès, R., Folcher, G. et Oumezine, M., Revue Phys. Appl. 20 (1985) 437.

[7] Nevot, L. and Croce, P., Revue Phys. Appl. 15 (1980) 761.

[8] Farges, J. P., J. Physique 46 (1985) 465.

Farges, J. P., J. Physique 46 (1985) 1249.

[9] Fischer, A., Sackmann, E., J. Physique 45 (1984) 517.

[10] Sarkar, M., Lando, J. B., Thin Solid Films 99, no 1/2/3 (1983). 